\title{
SEMIGROUP CONSTRUCTION ON POLYGONAL NUMBERS
}

\author{
Ahmet Emin \\ Ministry of National Education, 10662 Karesi, Ballkesir, Turkey \\ drahmetemin@gmail.com
}

\begin{abstract}
In this paper, some information about polygonal numbers are given. Also, a general binary operator that includes all polygonal numbers are given and it is investigated whether the algebraic structures defined with the general operator specify a semigroup or not.
\end{abstract}

Keywords: Polygonal numbers, binary operation, semigroup, monoid

\section{Introduction and motivation}

Polygonal numbers are positive integer numbers that can be denoted by regular geometric patterns. Starting from a point and continue to increase by the same common difference. If the common difference is one, then the geometric pattern is called triangular numbers. If it is two, then it becomes square numbers; if it is three, then it becomes pentagonal numbers. And so on. The theory of polygonal numbers does not belong to the central domains of mathematics, but the beauty of these numbers has attracted the attention of many scientists for thousands of years [1]. Many special numbers have been created by being inspired from polygonal numbers. Pythagoras triples, Perfect numbers, Mersenne numbers, Cullen numbers, Woodall numbers, Fermat numbers, Fibonacci numbers, Pell numbers, Lucas numbers, Thabit numbers, etc. are examples of such numbers.

Binary operators have played an important role in many algebraic structures. It takes various names according to the binary operation defined on algebraic structures. Groupoid, semigroup and monoid are some of them. Specifically, Sparavigna has given that it is a groupoid with binary operators defined on some polygonal numbers in [2-4]. By using similar methods as in these papers, we will give a general binary operator that includes all polygonal numbers. In addition, it will be investigated whether the algebraic structures defined with the general operator specify a semigroup or not. By the way, maybe other algebraic studies, such as the studies in [5-7], can be studied by other mathematicians with the binary operation defined on this new algebraic structure. 


\section{Preliminaries}

Definition 2.1 A groupoid $(S, *)$ is defined as a non-empty set $S$ on which a binary operation * (by which we mean a map $*: S \times S \rightarrow S$ ) is defined. In other words, groupoid is an algebraic structure on a set with a binary operator. The only restriction on the operator is closure. It means that applying the binary operator on two elements of given set $S$ returns with a value in which itself is a member of $S$. One can see more information in $[8,9]$. We say that $(S, *)$ is a semigroup if the operation $*$ is associative, that is to say, if, for all $x, y, z \in S$

$(x * y) * z=x *(y * z)$

A semigroup is an associative groupoid, a semigroup with an identity is called a monoid.

Definition 2.2 For $m=3,4,5, \ldots$ and $n \in \mathbb{N}$, nth $m$-gonal numbers formulas is as follows [1];

$S_{m}(n):=\frac{(m-2) n^{2}-(m-4) n}{2}$

Definition 2.3 Algebraically, for $n \in \mathbb{N}$ and $m \geq 3$, nth $m$-gonal number $S_{m}(n)$ is obtained as the sum of the first $n$ elements of the arithmetic progression $1,1+(m-2), 1+2(m-2), 1+$ $3(m-2), \ldots, 1+(n-1)(m-2)$. So, it holds [1];

$$
\begin{aligned}
S_{m}(n) & =1+(1+(m-2))+(1+2(m-2))+\cdots+(1+(n-1)(m-2)) \\
& =\frac{(m-2)\left(n^{2}-n\right)+2 n}{2} \\
& =\frac{(m-2) n^{2}-(m-4) n}{2} .
\end{aligned}
$$

Example 2.1 In particular, for $m=3,4,5,6,7,8$ and $n \in \mathbb{N}$, nth $m$-gonal numbers formulas are as follows;

$$
\begin{aligned}
& S_{3}(n)=\frac{n^{2}+n}{2}=\frac{n(n+1)}{2}, \\
& S_{4}(n)=\frac{2 n^{2}}{2}=n^{2} \\
& S_{5}(n)=\frac{3 n^{2}-n}{2}=\frac{n(3 n-1)}{2}, \\
& S_{6}(n)=\frac{4 n^{2}-2 n}{2}=\frac{2 n(n-1)}{2}=n(n-1), \\
& S_{7}(n)=\frac{5 n^{2}-3 n}{2}=\frac{n(5 n-3)}{2},
\end{aligned}
$$


$S_{8}(n)=\frac{6 n^{2}-4 n}{2}=\frac{2 n(3 n-2)}{2}=n(3 n-2)$.

Above expression implies the following recurrence formula for $m$-gonal numbers:

$S_{m}(n+1)=S_{m}(n)+(1+(m-2) n), S_{m}(1)=1$

In particular, we get

$S_{3}(n+1)=S_{3}(n)+(n+1)$,

$S_{4}(n+1)=S_{4}(n)+(2 n+1)$,

$S_{5}(n+1)=S_{5}(n)+(3 n+1)$,

$S_{6}(n+1)=S_{6}(n)+(4 n+1)$,

$S_{7}(n+1)=S_{7}(n)+(5 n+1)$,

$S_{8}(n+1)=S_{8}(n)+(6 n+1)$.

For many applications it is convenient to add the value $S_{m}(0)=0$ to the list.

Example 2.2 For $n=1,2,3,4,5$, and $m=3,4,5,6$ some polygonal numbers are as follows [10, A000217, A000290, A000326, A000384];

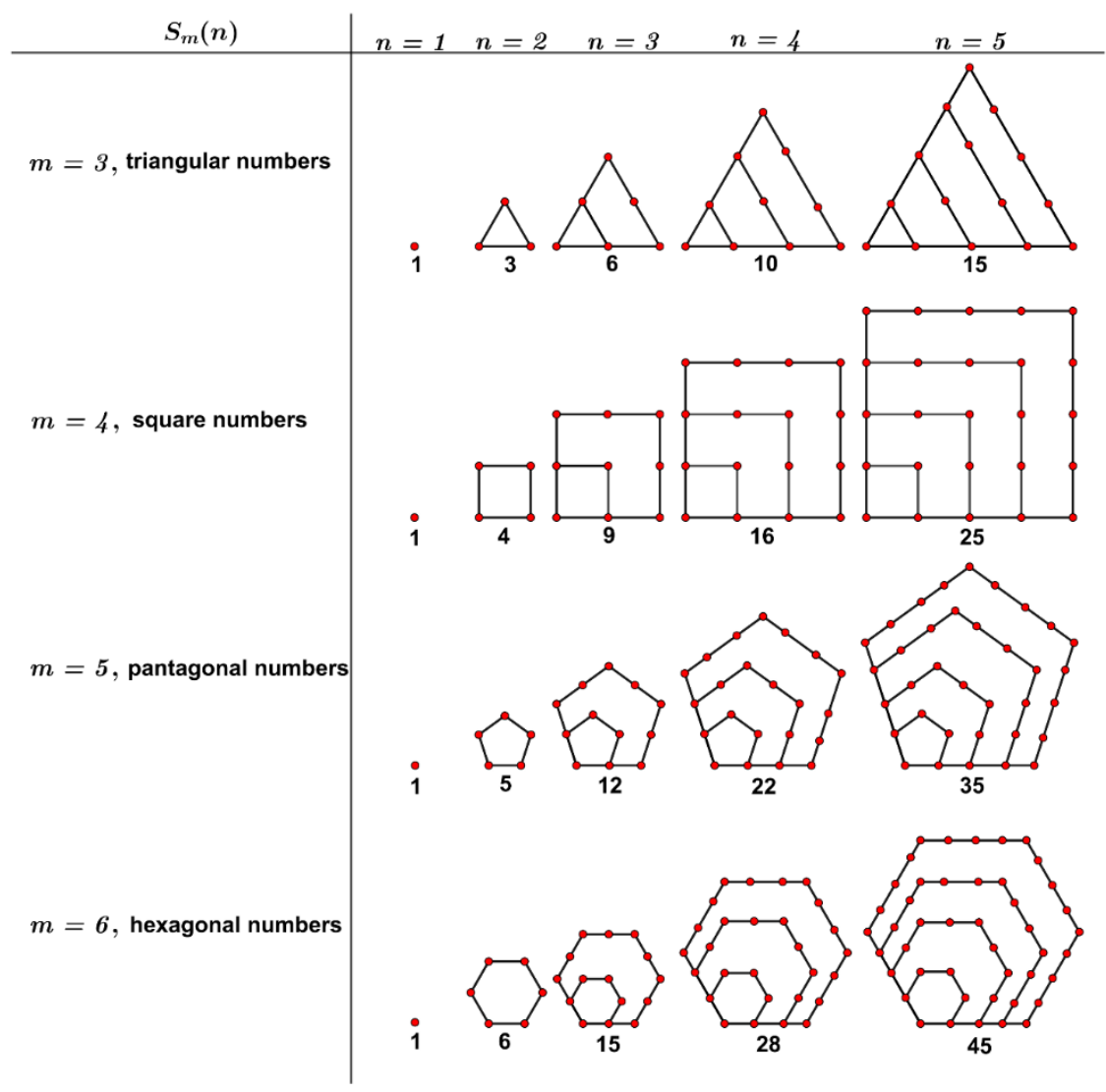

Figure 1. Some polygonal numbers 


\section{Semigroup construction on polygonal numbers}

In this section, we create a set consisting of elements of all polygonal numbers sequence $S_{m}(n)$. After that we obtain an algebraic structure by defining binary operation on the defined set. And finally, we give a theorem and a corollary that shows necessary conditions for this algebraic structure to be semigroup and monoid.

Before we can construct the theorem that yields the main result of this paper, we need to define a set and a binary operation on that set. So, let $M$ denote the sequence of numbers $S_{m}(n)$. That is, let

$$
\begin{aligned}
M=\{1, m, 3 m & -3,6 m-8,10 m-15, \ldots, \frac{(m-2) n^{2}-(m-4) n}{2} \\
& \left.+\left((2 m-4)\left(\frac{(m-2) n^{2}-(m-4) n}{2}\right)+\frac{(m-4)^{2}}{4}\right)^{\frac{1}{2}}+\frac{m-2}{2}, \ldots\right\} .
\end{aligned}
$$

Now we can find a binary operation of given set of $M$ Since

$$
\begin{aligned}
\left(S_{m}(n)+\frac{1}{8} \frac{(m-4)^{2}}{m-2}\right)^{\frac{1}{2}} & =\left(\frac{(m-2) n^{2}-(m-4) n}{2}+\frac{1}{8} \frac{(m-4)^{2}}{m-2}\right)^{\frac{1}{2}} \\
& =\left(\frac{(m-2) n^{2}}{2}-\frac{(m-4) n}{2}+\frac{1}{8} \frac{(m-4)^{2}}{m-2}\right)^{\frac{1}{2}} \\
& =\left(\frac{(m-2)}{2}\left(n-\frac{1}{2} \frac{m-4}{m-2}\right)^{2}\right)^{\frac{1}{2}} \\
& =\sqrt{\frac{(m-2)}{2}\left(n-\frac{m-4}{2(m-2)}\right) .}
\end{aligned}
$$

We define

$$
\begin{aligned}
& N_{n}=\left(S_{m}(n)+\frac{1}{8} \frac{(m-4)^{2}}{m-2}\right)^{\frac{1}{2}}=\sqrt{\frac{(m-2)}{2}}\left(n-\frac{m-4}{2(m-2)}\right), \\
& N_{k}=\left(S_{m}(k)+\frac{1}{8} \frac{(m-4)^{2}}{m-2}\right)^{\frac{1}{2}}=\sqrt{\frac{(m-2)}{2}\left(k-\frac{m-4}{2(m-2)}\right)} \\
& N_{n+k}=\left(S_{m}(n+k)+\frac{1}{8} \frac{(m-4)^{2}}{m-2}\right)^{\frac{1}{2}}=\sqrt{\frac{(m-2)}{2}}\left(n+k-\frac{m-4}{2(m-2)}\right) .
\end{aligned}
$$

We use $N_{n}$ for definition of binary operation.

$$
N_{n+k}=N_{n} * N_{k}=N_{n}+N_{k}+\frac{m-4}{2 \sqrt{2 m-4}}
$$




$$
\begin{aligned}
& =\sqrt{\frac{(m-2)}{2}}\left(n-\frac{m-4}{2(m-2)}\right)+\sqrt{\frac{(m-2)}{2}}\left(k-\frac{m-4}{2(m-2)}\right)+\frac{m-4}{2 \sqrt{2 m-4}} \\
& =\sqrt{\frac{(m-2)}{2}}\left(n+k-\frac{m-4}{2(m-2)}\right)+\frac{m-4}{2 \sqrt{2 m-4}}
\end{aligned}
$$

Therefore, we have the binary operation:

$$
\begin{aligned}
& \left(S_{m}(n+k)+\frac{1}{8} \frac{(m-4)^{2}}{m-2}\right)^{\frac{1}{2}}=N_{n+k}=N_{n} * N_{k}=N_{n}+N_{k}+\frac{m-4}{2 \sqrt{2 m-4}} \\
& =\left(S_{m}(n)+\frac{1}{8} \frac{(m-4)^{2}}{m-2}\right)^{\frac{1}{2}}+\left(S_{m}(k)+\frac{1}{8} \frac{(m-4)^{2}}{m-2}\right)^{\frac{1}{2}}+\frac{m-4}{2 \sqrt{2 m-4}} .
\end{aligned}
$$

As a result, from (11), we can rewrite the defined binary operation as follows:

$$
\begin{aligned}
S_{m}(n) * S_{m}(k) & =S_{m}(n+k) \\
& =S_{m}(n)+S_{m}(k)+\frac{1}{4} \frac{(m-4)^{2}}{m-2} \\
& +2\left(S_{m}(n)+\frac{1}{8} \frac{(m-4)^{2}}{m-2}\right)^{\frac{1}{2}}\left(S_{m}(k)+\frac{1}{8} \frac{(m-4)^{2}}{m-2}\right)^{\frac{1}{2}} \\
& +\frac{m-4}{2 \sqrt{2 m-4}}\left(S_{m}(n)+\frac{1}{8} \frac{(m-4)^{2}}{m-2}\right)^{\frac{1}{2}} \\
& +\frac{m-4}{2 \sqrt{2 m-4}}\left(S_{m}(k)+\frac{1}{8} \frac{(m-4)^{2}}{m-2}\right)^{\frac{1}{2}} .
\end{aligned}
$$

In the following theorem, we give a necessary condition for the algebraic structure $(M, *)$ to be a semigroup which is the main result of this paper.

Theorem 3.1 Let $M$ be the set of sequence of numbers $S_{m}(n)$, that is, let

$$
\begin{aligned}
M=\{1, m, 3 m & -3,6 m-8,10 m-15, \ldots, \frac{(m-2) n^{2}-(m-4) n}{2} \\
& \left.+\left((2 m-4)\left(\frac{(m-2) n^{2}-(m-4) n}{2}\right)+\frac{(m-4)^{2}}{4}\right)^{\frac{1}{2}}+\frac{m-2}{2}, \ldots\right\} .
\end{aligned}
$$

Also let $*$ a binary operation on $M$ such that

$$
\begin{aligned}
S_{m}(n) * S_{m}(k) & =S_{m}(n+k) \\
& =S_{m}(n)+S_{m}(k)+\frac{1}{4} \frac{(m-4)^{2}}{m-2}
\end{aligned}
$$




$$
\begin{aligned}
& +2\left(S_{m}(n)+\frac{1}{8} \frac{(m-4)^{2}}{m-2}\right)^{\frac{1}{2}}\left(S_{m}(k)+\frac{1}{8} \frac{(m-4)^{2}}{m-2}\right)^{\frac{1}{2}} \\
& +\frac{m-4}{2 \sqrt{2 m-4}}\left(S_{m}(n)+\frac{1}{8} \frac{(m-4)^{2}}{m-2}\right)^{\frac{1}{2}}+\frac{m-4}{2 \sqrt{2 m-4}}\left(S_{m}(k)+\frac{1}{8} \frac{(m-4)^{2}}{m-2}\right)^{\frac{1}{2}} .
\end{aligned}
$$

where $n, k \in \mathbb{N}$ and $m \geq 3$. Then the algebraic structure $(M, *)$ is a semigroup.

Proof. From binary operation $*$, we can have recursive relation for $k=1$ :

$$
\begin{aligned}
S_{m}(n) * S_{m}(1) & =S_{m}(n+1) \\
& =S_{m}(n)+S_{m}(1)+\frac{1}{4} \frac{(m-4)^{2}}{m-2} \\
& +2\left(S_{m}(n)+\frac{1}{8} \frac{(m-4)^{2}}{m-2}\right)^{\frac{1}{2}}\left(S_{m}(1)+\frac{1}{8} \frac{(m-4)^{2}}{m-2}\right)^{\frac{1}{2}} \\
& +\frac{m-4}{2 \sqrt{2 m-4}}\left(S_{m}(n)+\frac{1}{8} \frac{(m-4)^{2}}{m-2}\right)^{\frac{1}{2}}+\frac{m-4}{2 \sqrt{2 m-4}}\left(S_{m}(1)+\frac{1}{8} \frac{(m-4)^{2}}{m-2}\right)^{\frac{1}{2}} . \\
S_{m}(n+1)= & S_{m}(n) * S_{m}(1)=S_{m}(n)+\frac{m-2}{2}+\sqrt{2 m-4}\left(S_{m}(n)+\frac{1}{8} \frac{(m-4)^{2}}{m-2}\right)^{\frac{1}{2}}
\end{aligned}
$$

and so, we have

$$
S_{m}(n+1)=S_{m}(n)+\left((2 m-4) S_{m}(n)+\frac{(m-4)^{2}}{4}\right)^{\frac{1}{2}}+\frac{m-2}{2} .
$$

Starting from number $S_{m}(1)=1$, we have $m, 3 m-3,6 m-8,10 m-15,15 m-24, \ldots$, $\frac{(m-2) n^{2}-(m-4) n}{2}+\left((2 m-4)\left(\frac{(m-2) n^{2}-(m-4) n}{2}\right)+\frac{(m-4)^{2}}{4}\right)^{\frac{1}{2}}+\frac{m-2}{2}, \ldots$ which are the elements of the set of $M$. Indeed, for $m \geq 3$,

$$
\begin{aligned}
S_{m}(2) & =S_{m}(1)+\left((2 m-4) S_{m}(1)+\frac{(m-4)^{2}}{4}\right)^{\frac{1}{2}}+\frac{m-2}{2} \\
& =1+\left(2 m-4+\frac{(m-4)^{2}}{4}\right)^{\frac{1}{2}}+\frac{m-2}{2}=1+\frac{m}{2}+\frac{m-2}{2}=m \\
S_{m}(3) & =S_{m}(2)+\left((2 m-4) S_{m}(2)+\frac{(m-4)^{2}}{4}\right)^{\frac{1}{2}}+\frac{m-2}{2} \\
& =m+\left((2 m-4) m+\frac{(m-4)^{2}}{4}\right)^{\frac{1}{2}}+\frac{m-2}{2}=m+\frac{3 m-4}{2}+\frac{m-2}{2}=3 m-3 . \\
S_{m}(4) & =S_{m}(3)+\left((2 m-4) S_{m}(3)+\frac{(m-4)^{2}}{4}\right)^{\frac{1}{2}}+\frac{m-2}{2}
\end{aligned}
$$




$$
\begin{aligned}
& =3 m-3+\left((2 m-4)(3 m-3)+\frac{(m-4)^{2}}{4}\right)^{\frac{1}{2}}+\frac{m-2}{2} \\
& =3 m-3+\frac{5 m-8}{2}+\frac{m-2}{2}=6 m-8 . \\
S_{m}(5) & =S_{m}(4)+\left((2 m-4) S_{m}(4)+\frac{(m-4)^{2}}{4}\right)^{\frac{1}{2}}+\frac{m-2}{2} \\
& =6 m-8+\left((2 m-4)(6 m-8)+\frac{(m-4)^{2}}{4}\right)^{\frac{1}{2}}+\frac{m-2}{2} \\
& =6 m-8+\frac{7 m-12}{2}+\frac{m-2}{2}=10 m-15 .
\end{aligned}
$$

And so on. That means, the algebraic structure $(M, *)$ satisfies the properties of closure. Now we need to show that $(M, *)$ satisfies the properties of associativity. We know from the construction of binary operation $*$ that

$N_{n+k}=N_{n} * N_{k}=N_{n}+N_{k}+\frac{m-4}{2 \sqrt{2 m-4}}$, where $n, k \in \mathbb{N}, m \geq 3$ and

$N_{n}=\left(S_{m}(n)+\frac{1}{8} \frac{(m-4)^{2}}{m-2}\right)^{\frac{1}{2}}=\sqrt{\frac{m-2}{2}}\left(n-\frac{m-4}{2(m-2)}\right)$,

$N_{k}=\left(S_{m}(k)+\frac{1}{8} \frac{(m-4)^{2}}{m-2}\right)^{\frac{1}{2}}=\sqrt{\frac{m-2}{2}}\left(k-\frac{m-4}{2(m-2)}\right)$,

$N_{n+k}=\left(S_{m}(n+k)+\frac{1}{8} \frac{(m-4)^{2}}{m-2}\right)^{\frac{1}{2}}=\sqrt{\frac{m-2}{2}}\left(n+k-\frac{m-4}{2(m-2)}\right)$.

For $n, k, p \in \mathbb{N}$ and $m \geq 3$

$$
\begin{aligned}
\left(N_{n} * N_{k}\right) * N_{p} & =N_{n+k}+N_{p}+\frac{m-4}{2 \sqrt{2 m-4}} \\
& =\sqrt{\frac{m-2}{2}}\left(n+k-\frac{m-4}{2(m-2)}\right)+\sqrt{\frac{m-2}{2}}\left(p-\frac{m-4}{2(m-2)}\right)+\frac{m-4}{2 \sqrt{2 m-4}} \\
& =\sqrt{\frac{m-2}{2}}\left(n+k+p-\frac{m-4}{m-2}\right)+\frac{m-4}{2 \sqrt{2 m-4}}
\end{aligned}
$$

and

$$
\begin{aligned}
N_{n} *\left(N_{k} * N_{p}\right) & =N_{n}+N_{k+p}+\frac{m-4}{2 \sqrt{2 m-4}} \\
& =\sqrt{\frac{m-2}{2}}\left(n-\frac{m-4}{2(m-2)}\right)+\sqrt{\frac{m-2}{2}}\left(k+p-\frac{m-4}{2(m-2)}\right)+\frac{m-4}{2 \sqrt{2 m-4}}
\end{aligned}
$$




$$
=\sqrt{\frac{m-2}{2}}\left(n+k+p-\frac{m-4}{m-2}\right)+\frac{m-4}{2 \sqrt{2 m-4}} .
$$

So, with the results of equations (14) and (15), we obtain, $\left(N_{n} * N_{k}\right) * N_{p}=N_{n} *\left(N_{k} * N_{p}\right)$ which gives us that $(M, *)$ satisfies the properties of associativity. Hence the result.

As seen in Figure 1, polygonal numbers start from $S_{m}(1)=1$. However, in some studies, as you can see in [1, 10], polygonal numbers start from the number $S_{m}(0)=0$. Now by considering the start point as the number $S_{m}(0)=0$ and Theorem 3.1, we can give the following corollary which gives the conditions for $(M, *)$ to be a monoid.

Corollary 3.1 Let $M$ be the set of sequence of numbers $S_{m}(n)$ and let * a binary operation on $M$ (defined in (7)) such that,

$$
\begin{aligned}
S_{m}(n) * S_{m}(k) & =S_{m}(n+k) \\
& =S_{m}(n)+S_{m}(k)+\frac{1}{4} \frac{(m-4)^{2}}{m-2} \\
& +2\left(S_{m}(n)+\frac{1}{8} \frac{(m-4)^{2}}{m-2}\right)^{\frac{1}{2}}\left(S_{m}(k)+\frac{1}{8} \frac{(m-4)^{2}}{m-2}\right)^{\frac{1}{2}} \\
& +\frac{m-4}{2 \sqrt{2 m-4}}\left(S_{m}(n)+\frac{1}{8} \frac{(m-4)^{2}}{m-2}\right)^{\frac{1}{2}}+\frac{m-4}{2 \sqrt{2 m-4}}\left(S_{m}(k)+\frac{1}{8} \frac{(m-4)^{2}}{m-2}\right)^{\frac{1}{2}} .
\end{aligned}
$$

where $n, k \in \mathbb{N}$ and $m \geq 3$. If $S_{m}(0)=0 \in M$, then the algebraic structure $(M, *)$ is a monoid.

Proof. In Theorem 2.1, we have proved that $(M, *)$ is a semigroup. To show that the algebraic structure $(M, *)$ is a monoid, it must be shown that it has an identity element. Let $S_{m}(0)=0 \in$ $M$, then, from the binary operation $*$ we have,

$S_{m}(n) * S_{m}(0)=S_{m}(n+0)=S_{m}(n)$ and $S_{m}(0) * S_{m}(n)=S_{m}(0+n)=S_{m}(n)$.

Thus, we have, $S_{m}(n) * S_{m}(0)=S_{m}(0) * S_{m}(n)=S_{m}(n)$ which gives us that $(M, *)$ satisfies the properties of identity. Hence the result.

Example 3.1 [10, A000290] known as square numbers. Square numbers are integer having following form:

$S_{4}(n)=1+3+5+\ldots+2 n-1=n^{2}$.

Let $A$ denote the sequence of numbers $S_{4}(n)$. That is, let $A=\{1,4,9,16,25,36,49,64,81$, $100,121,144,169,225,256,289,324,361,400,441,484, \ldots\}$. Now we can find a binary operation of given set of $A$. Since

$\left(S_{4}(n)\right)^{\frac{1}{2}}=\left(n^{2}\right)^{\frac{1}{2}}=n$

we define: 
$S_{n}=\left(S_{4}(n)\right)^{\frac{1}{2}}=\left(n^{2}\right)^{\frac{1}{2}}=n$

$S_{k}=\left(S_{4}(k)\right)^{\frac{1}{2}}=\left(k^{2}\right)^{\frac{1}{2}}=k$

$S_{n+k}=\left(S_{4}(n+k)\right)^{\frac{1}{2}}=\left((n+k)^{2}\right)^{\frac{1}{2}}=n+k$.

We use $S_{n}$ for definition of binary operation:

$$
\begin{aligned}
S_{n+k} & =S_{n} * S_{k}=S_{4}(n+k)=\left(S_{4}(n)\right)^{\frac{1}{2}}+\left(S_{4}(k)\right)^{\frac{1}{2}}+2\left(S_{4}(n)\right)^{\frac{1}{2}}\left(S_{4}(k)\right)^{\frac{1}{2}} \\
& =\left(n^{2}\right)^{\frac{1}{2}}+\left(k^{2}\right)^{\frac{1}{2}}=n+k .
\end{aligned}
$$

As a result, we can rewrite the defined binary operation as follows:

$S_{4}(n) * S_{4}(k)=S_{4}(n+k)=S_{4}(n)+S_{4}(k)+2\left(S_{4}(n)\right)^{\frac{1}{2}}\left(S_{4}(k)\right)^{\frac{1}{2}}$.

From binary operation $*$, we can have recursive relation for $k=1$ :

$S_{4}(n+1)=S_{4}(n) * S_{4}(1)=S_{4}(n)+2\left(S_{4}(n)\right)^{\frac{1}{2}}+1$.

And so, we obtain

$S_{4}(n+1)=S_{4}(n)+2\left(S_{4}(n)\right)^{\frac{1}{2}}+1$.

Starting from number $S_{4}(1)=1$, we have 4, 9, 16, 25, 36, 49, 64, 81, 100, 121, 144, 169, 196, 225, 256, 289, 324,361, 400, 441, 484, 529, ... which are the elements of the set of $A$. From Theorem 3.1, one can say that the algebraic structure $(A, *)$ is a semigroup. Also, if the $S_{4}(0)=$ $0 \in A$ then the algebraic structure $(A, *)$ is a monoid from Corollary 3.1.

Example 3.2 [10, A000384] known as hexagonal numbers. Hexagonal numbers are integer having following form:

$S_{6}(n)=1+5+9+\ldots+4 n-3=n(2 n-1)$.

Let $B$ denote the sequence of numbers $S_{6}(n)$. That is, let $B=\{1,6,15,28,45,66,91,120$, $153,190,231,276,325,378,435,496,561,630,703,780, \ldots\}$. Now we can find a binary operation of given set of $B$. Since

$\left(S_{6}(n)+\frac{1}{8}\right)^{\frac{1}{2}}=\left(\frac{1}{2} n(4 n-2)+\frac{1}{8}\right)^{\frac{1}{2}}=\left(2\left(n-\frac{1}{4}\right)^{2}\right)^{\frac{1}{2}}=\sqrt{2}\left(n-\frac{1}{4}\right)$.

we define:

$$
\begin{aligned}
& H_{n}=\left(S_{6}(n)+\frac{1}{8}\right)^{\frac{1}{2}}=\sqrt{2}\left(n-\frac{1}{4}\right) \\
& H_{k}=\left(S_{6}(k)+\frac{1}{8}\right)^{\frac{1}{2}}=\sqrt{2}\left(k-\frac{1}{4}\right)
\end{aligned}
$$


$H_{n+k}=\left(S_{6}(n+k)+\frac{1}{8}\right)^{\frac{1}{2}}=\sqrt{2}\left(n+k-\frac{1}{4}\right)$.

We use $H_{n}$ for definition of binary operation:

$H_{n+k}=H_{n} * H_{k}=H_{n}+H_{k}+\frac{1}{2 \sqrt{2}}=\sqrt{2}\left(n-\frac{1}{4}\right)+\sqrt{2}\left(k-\frac{1}{4}\right)+\frac{1}{2 \sqrt{2}}=\sqrt{2}\left(n+k-\frac{1}{4}\right)$.

As a result, we can rewrite the defined binary operation as follows:

$$
\begin{aligned}
S_{6}(n) * S_{6}(k) & =S_{6}(n+k) \\
& =S_{6}(n)+S_{6}(k)+\frac{1}{4} \\
& +2\left(S_{6}(n)+\frac{1}{8}\right)^{\frac{1}{2}}\left(S_{6}(k)+\frac{1}{8}\right)^{\frac{1}{2}}+\frac{1}{\sqrt{2}}\left(S_{6}(n)+\frac{1}{8}\right)^{\frac{1}{2}}+\frac{1}{\sqrt{2}}\left(S_{6}(k)+\frac{1}{8}\right)^{\frac{1}{2}} .
\end{aligned}
$$

From binary operation $*$, we can have recursive relation for $k=1$ :

$S_{6}(n+1)=S_{6}(n) * S_{6}(1)=S_{6}(n)+2+2 \sqrt{2}\left(S_{6}(n)+\frac{1}{8}\right)^{\frac{1}{2}}$.

And so, we obtain

$S_{6}(n+1)=S_{6}(n)+\left(8 S_{6}(n)+1\right)^{\frac{1}{2}}+2$.

Starting from number $S_{6}(1)=1$, we have $6,15,28,45,66,91,120,153,190,231,276,325$, 378, 435, 496, 561, 630, 703, ...which are the elements of the set of $B$. From Theorem 3.1, one can say that the algebraic structure $(B, *)$ is a semigroup. Also, if the $S_{6}(0)=0 \in B$, then the algebraic structure $(B, *)$ is a monoid from Corollary 3.1.

\section{References}

[1] Deza, E., Deza, M. M., "Figurate Numbers”, World Scientific Publishing, Singapore, (2012).

[2] Sparavigna, A. C., "On a generalized sum of the Mersenne Numbers", Zenodo. http://doi.org/10.5281/zenodo.1250048, (2018).

[3] Sparavigna, A. C., "Groupoids of OEIS A093112 and A093069 Numbers (oblong and odd square numbers)”, Zenodo. http://doi.org/10.5281/zenodo.3247003, (2019).

[4] Sparavigna, A. C., "Groupoids of OEIS A003154 Numbers (star numbers or centered dodecagonal numbers)”, Zenodo. http://doi.org/10.5281/zenodo.3387054, (2019).

[5] Ateş, F., Emin, A., "Some New Results on the Orthodox, Strongly $\pi$ - Inverse and $\pi$ Regularity of Some Monoids”, Bulletin of The Society of Mathematicians Banja Luka, 11(3) (2021) : 463-472.

[6] Ateş, F., "Some new monoid and group constructions under semidirect products", Ars. Combinatoria, 91 (2009) : 203-218.

[7] Emin, A., Ateş, F., “A new monoid construction under crossed products”, Journal of Inequalities and Applications, 244(1) (2013). 
[8] Howie, J. M., “Fundamentals of Semigroup Theory”, No 12, Clarendon Press, Oxford, (1995).

[9] Stover, C., Weisstein, E. W., “Groupoid” Retrieved in January, 30, 2021 from http://mathworld.wolfram.com/Groupoid.html, (2021).

[10] The On-Line Encyclopedia of Integer Sequences, OEIS Foundation Inc., http://oeis.org, (2021). 